\title{
Uptake of the fluorescent probe FM4-64 by hyphae and haemolymph-derived in vivo hyphal bodies of the entomopathogenic fungus Beauveria bassiana
}

Correspondence

Nemat O. Keyhani

keyhani@ufl.edu

Received 12 March 2009

Revised 26 May 2009

Accepted 15 June 2009

\author{
Michael W. Lewis, Ines V. Robalino and Nemat O. Keyhani \\ Department of Microbiology and Cell Science, University of Florida, Gainesville, FL 32611, USA
}

\begin{abstract}
The entomopathogenic fungus Beauveria bassiana is under intensive study as a pest biological control agent. $B$. bassiana produces several distinct single-cell types that include aerial conidia, in vitro blastospores and submerged conidia. Under appropriate nutrient conditions these cells can elaborate germ tubes that form hyphae, which in turn lead to the formation of a fungal mycelium. In addition, $B$. bassiana displays a dimorphic transition, producing in vivo specific yeast-like hyphal bodies during growth in the arthropod haemolymph. The amphiphilic styryl dye FM4-64 was used to investigate internalization and morphological features of in vitro and in vivo insect haemolymphderived $B$. bassiana cells. In vitro blastospores and submerged conidia displayed a punctate pattern of internal labelling, whereas aerial conidia failed to internalize the dye under the conditions tested. FM4-64 was also taken up into both apical and subapical compartments of living hyphae in a time-dependent manner, with clearly observable vesicle labelling. Internalization, where occurring, was reversibly disrupted by lowering the temperature of the assay or by treatment with azide/fluoride and latrunculin A. Treatment with cytochalasin D and monensin also caused abnormal vesicle trafficking, although some staining of vesicles was noted. Fungal cells derived from infected Heliothis virescens haemolymph (in vivo cells) actively internalized FM4-64. The in vivo blastospores or hyphal bodies displayed bright membrane and internal vesicle staining, although diffuse staining of internal structures was also visible. These results suggest active uptake by different developmental stages of $B$. bassiana, including haemolymph-derived cells that can evade the insect immune system.
\end{abstract}

\section{INTRODUCTION}

Insect pathogenic fungi, such as the ascomycete Beauveria bassiana (teleomorph Cordyceps bassiana) hold tremendous potential for use as pest biological control agents and as alternatives to chemical pesticides (McCoy, 1990; Samson et al., 1988). As such, B. bassiana is a ubiquitously distributed entomopathogenic fungus that displays a broad host range towards a diverse number of insect species (Inglis et al., 2001; Rehner \& Buckley, 2005). Strains of B. bassiana have been selected for control of insects and other arthropods that act as disease vectors, including mosquitoes and ticks (Kirkland et al., 2004; Scholte et al., 2004), nuisance and crop pests such as house and fruit flies (Dimbi et al., 2003; Lecuona et al., 2005), and even ecologically hazardous, invading crop pests such as locusts,

Abbreviations: CDCFDA, 5,6-carboxy-2',7'-dichlorofluorescein diacetate; DAPI, 4',6-diamidino-2-phenylindole; DIC, differential interference contrast; LYCH, Lucifer Yellow carbohydrazide; PI, propidium iodide.

A supplementary figure, showing FM4-64 staining of Manduca sexta haemolymph-derived in vivo hyphal bodies, is available with the online version of this paper. grasshoppers and termites (Kassa et al., 2004; Lomer et al., 2001).

For broad-host-range pathogens such as B. bassiana, the interaction between the pathogen and the host is mediated by the fungal cell wall, which has the ability to respond dynamically to surface cues and nutrient availability (Holder \& Keyhani, 2005; Holder et al., 2007). Internalization of external environmental nutrients and other molecules along with membrane protein and lipid turnover, via endocytosis or other mechanisms, represent critical processes required for growth and pathogenesis. Molecules internalized by endocytic vesicles are transported to endosomal compartments, from which they can be recycled back to membranes or sent to degradative organelles such as lysosomes or vacuoles. Endocytosis has been well characterized in mammalian, yeast and plant cells by both biochemical and genetic dissection (Ayscough, 2005; Gurunathan et al., 2002; Kaksonen et al., 2003; Murphy et al., 2005; Smythe \& Ayscough, 2006). Although there is substantial evidence that endocytosis occurs in (some) filamentous fungi, the issue is not without controversy (Read \& Kalkman, 2003; Torralba \& Heath, 2002). Methods for measuring uptake and/or the 
occurrence of endocytosis in living cells typically involve the use of fluorescent molecules such as Lucifer Yellow carbohydrazide (LYCH), FITC-dextran and the lipophilic styryl dye FM4-64, whose internalization can be imaged in real-time using conventional fluorescence or confocal microscopy (Cole et al., 1997; Torralba \& Heath, 2002; Vida \& Emr, 1995). Although internalization of LYCH and FITC-dextran has been reported in germinating conidia of the rice blast fungus Magnaporthe grisea, these compounds do not appear to be taken up by hyphae of a number of filamentous fungi (Atkinson et al., 2002; Read \& Kalkman, 2003). In contrast, FM4-64 is rapidly internalized by a broad range of fungal species, including Aspergillus nidulans, Botrytis cinerea, Neurospora crassa, Sclerotinia sclerotiorum and M. grisea amongst others, and has become the tool of choice for analysing uptake and vesicle trafficking in filamentous fungi (Fischer-Parton et al., 2000; Hickey et al., 2002; Penalva, 2005; Read \& Kalkman, 2003). Endocytosis has been demonstrated to be important for cell polarity and apical membrane recycling in Aspergillus oryzae as well as essential for pathogenic development in the plant pathogen Ustilago maydis (Fuchs et al., 2006; Higuchi et al., 2009). Preferential localization of the endocytic machinery to subapical rings in growing hyphae of $A$. nidulans has been used to suggest that the spatial coupling of apical secretion and subapical endocytosis may underlie hyphal growth (Araujo-Bazan et al., 2008). Although these studies have revealed a dynamic interplay between exocytosis, endocytosis and the cell cytoskeleton in the development of hyphal growth in filamentous fungi, little is known concerning these processes in fungi that can produce discrete non-filamentous single-cell stages.

B. bassiana can produce at least three distinct single-cell forms in vitro, including aerial conidia, blastospores and submerged conidia, that can be isolated in near-homogeneous populations from agar plates, rich broth and nutrientlimited submerged cultures, respectively (Cho et al., 2006b). Fungal hyphae penetrate the insect cuticle, reaching the haemocoel, whereupon they differentiate into single cells termed in vivo blastospores and/or hyphal bodies that proliferate on the nutrients available in the host haemolymph (Pendland et al., 1993; Tartar \& Boucias, 2004). In the accompanying paper we describe the carbohydrate epitopes elaborated during different developmental stages of B. bassiana (Wanchoo et al., 2009). In this study, we have used FM4-64 to investigate internalization and morphological features of in vitro- and in vivo-derived $B$. bassiana cells. The dye stained the outer membranes of aerial conidia, in vitro blastospores and submerged conidia. Uptake occurred in both in vitro blastospores and submerged conidia, but not aerial conidia. FM4-64 was taken up into both apical and subapical compartments of germinated conidia and hyphae in a time-dependent manner. Internalization was reversibly disrupted by lowering the temperature of the assay or by treatment with azide/fluoride, cytochalasin D, monensin and latrunculin. Analysis of fungal cells recovered from insect haemolymph revealed that in vivo blastospores or hyphal bodies were significantly larger than their in vitro counterparts, and also actively internalized the FM4-64 dye.

\section{METHODS}

Chemical reagents and fungal material. FM4-64 and LYCH were obtained from Molecular Probes-Invitrogen. All other reagents and chemicals were obtained from either Sigma-Aldrich or Fisher Scientific. B. bassiana (ATCC 90517) was routinely grown on potato dextrose agar (PDA). Plates were incubated at $26{ }^{\circ} \mathrm{C}$ for $10-14$ days and aerial conidia were harvested by flooding the plate with sterile distilled $\mathrm{H}_{2} \mathrm{O}$. Conidial suspensions were filtered through a single layer of Miracloth and final spore concentrations were determined by direct counting using a haemocytometer. Blastospores were produced in Sabouraud dextrose $+0.5 \%$ yeast extract (SDY) liquid broth cultures using conidia harvested from plates to a final concentration of $0.5-5 \times 10^{5}$ conidia $\mathrm{ml}^{-1}$ as the inoculum. Cultures were grown for 3-4 days at $26{ }^{\circ} \mathrm{C}$ with aeration. Cultures were filtered through glass wool or Miracloth to remove mycelia, and the concentration of blastospores was determined by direct counting. Submerged conidia were produced in TKI broth using fructose as the carbon source, as described by Thomas et al. (1987). For all (in vitro) single-cell types, Miracloth- or glass wool-filtered cell suspensions were harvested by centrifugation $\left(7000 \mathrm{~g}, 10 \mathrm{~min}, 4^{\circ} \mathrm{C}\right.$ ), washed once with medium, and resuspended to the desired concentration (typically $10^{7}-10^{8}$ cells $\mathrm{ml}^{-1}$ ). Hyphae were produced by inoculation of SDY medium with aerial conidia and cultures were allowed to grow for $12-24 \mathrm{~h}$ at $26{ }^{\circ} \mathrm{C}$ before use. The production of in vivo fungal cells was performed essentially as described by Tartar \& Boucias (2004). Two different insect systems were used to isolate B. bassiana-derived in vivo cells. Briefly, the tobacco hornworm Manduca sexta accessed from laboratory colonies and the tobacco budworm Heliothis virescens were used as hosts to isolate in vivo fungal cells. Third to fifth larval instars reared on an artificial diet at $25^{\circ} \mathrm{C}$ were challenged by injection of either (in vitro) blastospores or aerial conidia $\left(2-5 \times 10^{4}\right.$ cells per larva) into the haemocoel. Injected larvae were incubated for 68-72 $\mathrm{h}$ at $25^{\circ} \mathrm{C}$, after which individual larvae were injected with $0.5 \mathrm{ml}$ anticoagulant solution $(0.14 \mathrm{M} \mathrm{NaCl}, 0.1 \mathrm{M}$ glucose, $26 \mathrm{mM}$ citric acid, $30 \mathrm{mM}$ trisodium citrate, $10 \mathrm{mM}$ EDTA, $\mathrm{pH}$ 4.6; also inhibits the phenoloxidase cascade) and bled. In vivo fungal cells were separated from insect haemocytes by 25-50 \% step Centricoll gradient centrifugation as a pellet at the bottom of the centrifuge tube. Pellets were washed free of gradient material into the desired uptake buffer and used immediately. Collection of haemolymph, centrifugation and subsequent washing steps were all performed at $4{ }^{\circ} \mathrm{C}$. Viability of the cells was determined by observing the growth of the cells when incubated in Sabouraud dextrose agar (SAB) medium (8-12 h) and by propidium iodide (PI) exclusion.

Treatment of cells with fluorescent probes. Aerial conidia, submerged conidia and blastospores were harvested by centrifugation (7000 $\mathrm{g}, 10 \mathrm{~min}, 4^{\circ} \mathrm{C}$ ) and washed once into the desired solution,

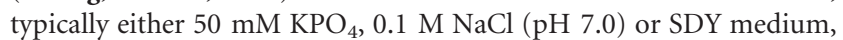
as indicated. Germinated conidia were used directly from the growth culture or diluted into PBS or fresh medium (1:2-4) for use in dyelabelling experiments. Alternatively, conidia were placed onto coverslips containing medium and allowed to germinate on the coverslip overnight before use. Two low-molecular-mass membrane-impermeant probes, FM4-64 (1 mM stock solution in EtOH) and LYCH (1 mM stock in aqueous solution), were employed. Several different methods of dye loading were employed. (1) Room temperature loading, no wash. Briefly, dye was added to cell suspensions to a final concentration of $2-10 \mu \mathrm{M}$, and samples $(50 \mu \mathrm{l})$ were then taken and placed on cleaned microscope slides with coverslips and examined 
over a time-course (up to $2 \mathrm{~h}$ ) in the dye solution. (2) Room temperature loading + wash. Briefly, dye was added to cell suspensions to a final concentration of $2-10 \mu \mathrm{M}$. Samples (typically $0.1 \mathrm{ml}$ ) were loaded with the dye for $15 \mathrm{~min}$, after which the cells were collected by centrifugation $(7000 \mathrm{~g}, 5 \mathrm{~min})$ and resuspension of the cells in fresh medium without the dye $(0.1 \mathrm{ml}) .(3,4)$ Cold loading \pm wash. Samples were incubated in the presence of the dye on ice for either 15 or $30 \mathrm{~min}$ and visualized at room temperature with or without a subsequent wash into medium without the dye. Cell samples double-labelled using the vacuolar dye 5,6-carboxy-2',7'-dichlorofluorescein diacetate (CDCFDA) were first incubated with FM4-64 for 30 min (room temperature, no wash) prior to the addition of $3 \mu \mathrm{M}$ (final concentration) CDCFDA. Cell samples double-labelled using 4',6diamidino-2-phenylindole (DAPI) nuclear stain were first loaded with FM4-64 for $45 \mathrm{~min}$ at room temperature, followed by fixation of the cells using $2 \%$ paraformaldehyde in PBS for $20 \mathrm{~min}$. Samples were then harvested by centrifugation $(7000 \mathrm{~g}, 5 \mathrm{~min})$ and resuspended in $100 \mu \mathrm{l}$ of a $1: 1000$ dilution of stock DAPI solution $\left(1 \mathrm{mg} \mathrm{ml}^{-1}\right)$ in PBS and incubated for 5-10 min before imaging.

Inhibitor treatments. The effect of various inhibitors was tested by pretreating samples with the indicated compound for $15 \mathrm{~min}$ prior to the addition of FM4-64. Experiments were performed using the various different dye-loading protocols described above. In some experiments, cells were treated with the inhibitor for $2 \mathrm{~h}$, after which the inhibitor solution was removed, and the cells were washed once and then resuspended in fresh medium lacking the inhibitor (or dye). Cells were then allowed to recover for up to $4 \mathrm{~h}$, after which the dye was added to the cells and the cells were examined. The final concentrations of the chemical compounds tested were as follows: $5 \mathrm{mM}$ azide $+5 \mathrm{mM}$ fluoride (cocktail containing both compounds), $5 \mu \mathrm{M}$ cytochalasin $\mathrm{D}, 50 \mu \mathrm{M}$ latrunculin $\mathrm{A}$ and $5 \mu \mathrm{M}$ monensin.

Microscopy. Mounted slides were observed using a PASCAL LSM 5 confocal microscope fitted with Nomarski differential interference contrast (DIC) optics and an LP560 filter $\left(\mathrm{Ex}_{543} / \mathrm{Em}_{560}\right)$ or LP505 filter $\left(\mathrm{Ex}_{488} / \mathrm{Em}_{518}\right)$ for FM4-64 and CDCFDA visualization, respectively. Samples co-stained with DAPI were visualized using an Olympus BH2-RFCA fluorescence microscope with a Qimaging Retiga 2000R camera and rhodamine (BP545, $\mathrm{Ex}_{470-550} / \mathrm{Em}_{590}$ ) and UV $\left(\mathrm{Ex}_{365} / \mathrm{Em}_{460}\right)$ filter sets. Observations were made on the singlecelled propagules and on the tip and penultimate cells of growing hyphae, with basal cells also examined. Viability of the single-celled propagules was confirmed by taking aliquots and determining the percentage germinating after overnight incubation in media. Viability of hyphae was confirmed by general appearance, presence of cytoplasmic streaming and observation of continued tip elongation and growth, as well as by PI exclusion. Images were recorded with a real-time digital-imaging set-up, and were arranged by Adobe Photoshop or similar software. At least 50 individual cells were examined for each experiment and all experiments were performed using at least three independent batches of cells. Quantification of FM4-64 intensity was performed using ImageJ pixelation software with Threshhold Colour and Color Counter plugins. An estimate of the percentage internalization was made by determining the ratio between the membrane-only signal, obtained by blacking out the internal signal in Photoshop, and the total fluorescent signal.

\section{RESULTS}

\section{Uptake of FM4-64 into discreet single-cell developmental stages and growing hyphae}

The uptake of FM4-64 into B. bassiana aerial conidia, in vitro blastospores and submerged conidia was investigated.
The purity of each single-cell type was determined microscopically and only cultures $>90 \%$ homogeneous were used for further study. No internalization of FM4-64 was observed by aerial conidia, even over a time-course of several hours in the presence or absence of growth medium (SDY), although the outer membrane was brightly labelled (Fig. 1a). In contrast, both in vitro blastospores and submerged conidia internalized the dye when washed into phosphate buffer, and a punctate pattern of internal staining could readily be seen (Fig. 1b, c). Experiments performed in medium gave essentially the same results,
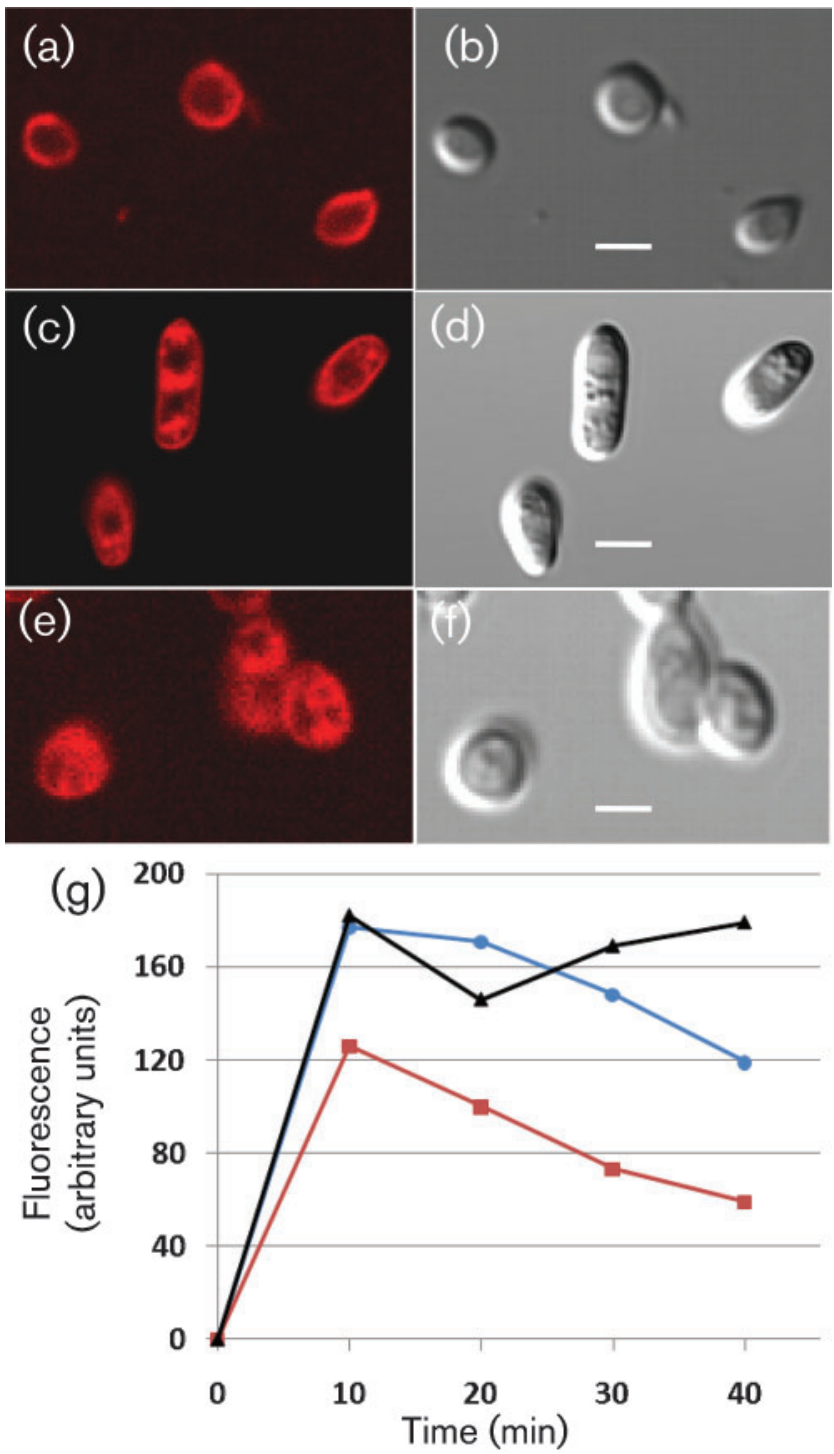

Fig. 1. FM4-64 staining of in vitro cells using the cold-load, nowash protocol. (a, c, e) Fluorescent images, (b, d, f) DIC images. Aerial conidia $(a, b)$, in vitro blastospores (c, d) and submerged conidia (e, f) incubated with $10 \mu \mathrm{M}$ FM4-64. Bars, $2.2 \mu \mathrm{m}$. (g) Quantification of FM4-64 signals from aerial conidia (black), blastospores (blue) and submerged conidia (red) treated using the room temperature, no-wash protocol. 
although the staining appeared to be more intense, but with a higher background visible (possibly due to interactions between the dye and medium components). Addition of glucose to the phosphate buffer during labelling experiments gave essentially the same results (data not shown). In order to determine the kinetics of FM4-64 staining, a room temperature, no-wash protocol was employed and samples were examined over the timecourse of the experiment, in which the signal was quantified as described in Methods. All three cell types showed an almost immediate strong staining (mainly of the outer membranes) that was stable over the time-course of the experiment, although some loss of signal, particularly for the submerged conidia, was observed (Fig. 1g). In contrast to the FM4-64 results, treatment of aerial conidia, in vitro blastospores and submerged conidia with $\mathrm{LYCH}$ resulted in outer membrane staining but no internalization under any of the conditions tested.

Addition of FM4-64 to germinated aerial conidia (germlings), without subsequent washing away of the dye, resulted in immediate and bright staining of germ tubes, hyphae, septa and the basal conidial cell (Fig. 2). In several instances, however, the basal conidial cell failed to stain with FM4-64, whereas the emerging germ tube was brightly stained. Staining of vesicles within hyphae was visible within 10-15 min of addition of FM4-64 (Fig. 2). Staining progressed over time from the membrane to small punctate spots (arrows in Fig. 2c- $\mathrm{f}$ ) to series of small and larger (presumed) vesicles (arrows in Fig. 2h-k) that could be
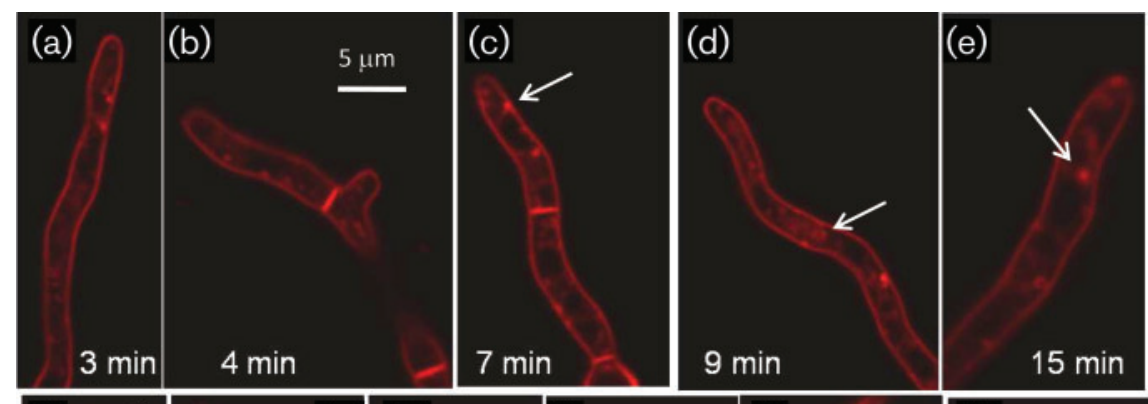

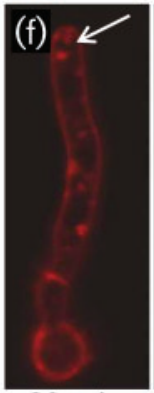

$20 \mathrm{~min}$

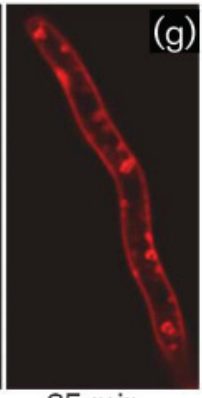

25 min

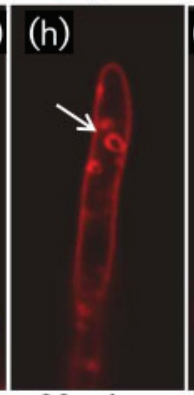

$30 \mathrm{~min}$

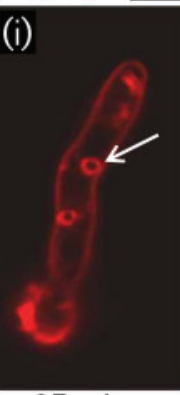

$35 \mathrm{~min}$

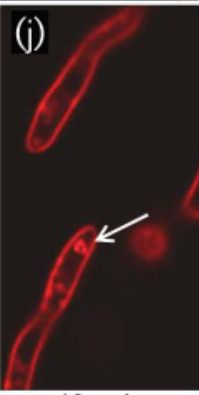

$40 \mathrm{~min}$

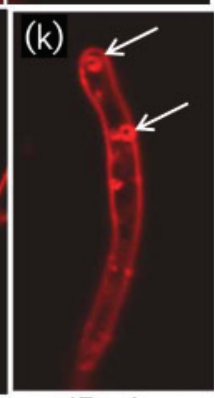

$45 \mathrm{~min}$

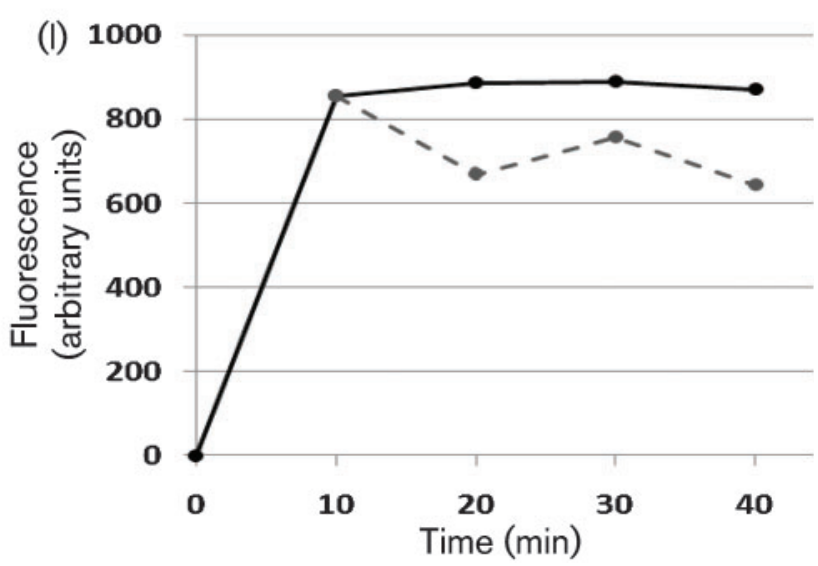

Fig. 2. FM4-64 uptake by B. bassiana hyphae. Time-course of room temperature load and visualization of FM4-64 in growing hyphae derived from aerial conidia $(a-k)$. The progression from the visualization of small punctate structures (presumed vesicles) to larger membrane-labelled vesicles/vacuoles is illustrated by the white arrows. Bar, $5.0 \mu$ m. (I) Quantification of the FM4-64 signal over time, showing total fluorescence intensity (solid line) and the membrane-only signal (dashed line). 
discerned in the hyphae. Hyphae maintained in growth medium or PBS supplemented with glucose gave essentially the same results. In several instances, a tubular reticular network was visible in the growing hyphae. Quantification of the FM4-64 signal revealed rapid membrane staining and internalization that was stable over time (Fig. 2l). An estimate of the percentage internalization was obtained by quantification of the total FM4-64 and membrane-only signals, and this ranged from 15 to $25 \%$ of the total fluorescence intensity observed.

Experiments performed using the cold-load followed by washing away of the dye (i.e. load and chase) before visualization resulted in less outer-membrane (FM4-64) signal, with staining of internal small and larger vesicles clearly visible (Fig. 3). Dye staining proceeded from cortical punctate structures (Fig. 3a, arrows) to large vesicles (Fig. 3b-f), including some found in the basal conidium. In several instances, a diffuse internal staining was also noted, that may represent a thread-like material involving mitochondrial membranes, as described in Aspergillus (Penalva, 2005). The larger circular vesicles whose membranes were stained by FM4-64 were identified as vacuoles by dual-label experiments performed using the

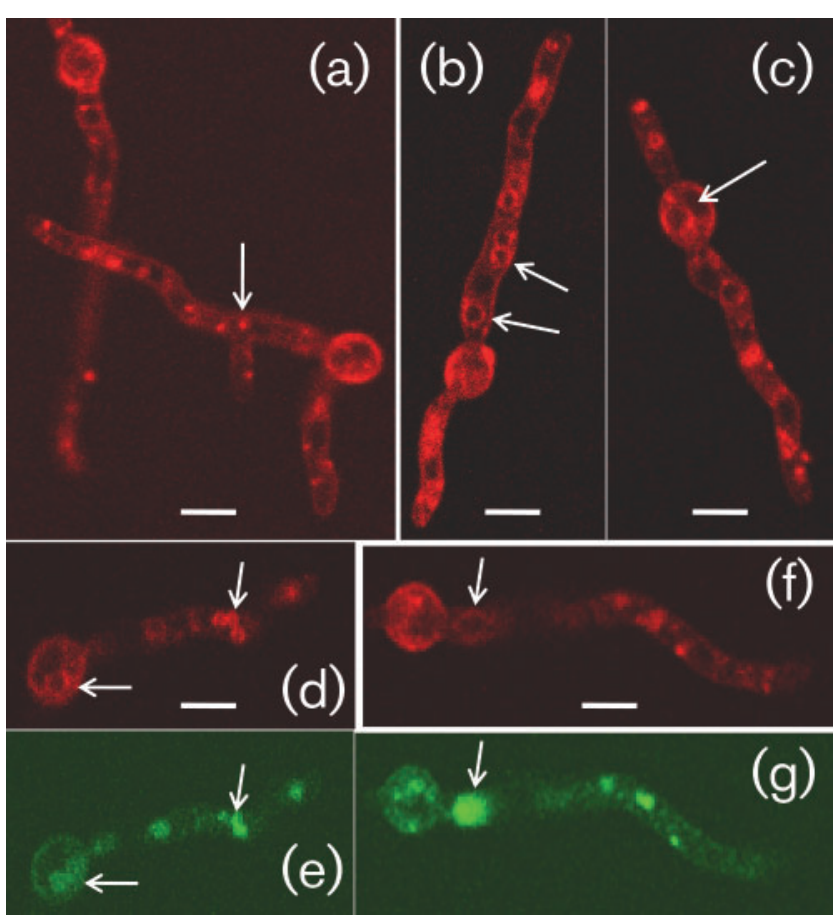

Fig. 3. Load and chase analysis of FM4-64 uptake. Time-course of FM4-64 internalization after 30 min cold $\left(4{ }^{\circ} \mathrm{C}\right)$, followed by wash into fresh buffer (chase), $5 \mathrm{~min}$ (a), $15 \mathrm{~min}$ (b) and $30 \mathrm{~min}$ (c) post-chase. Dual labelling using CDCFDA, 35 min FM4-64 (chased), 5 min CDCFDA (d, e), and 45 min FM4-64 (chased), 15 min CDCFDA ( $f, g)$. Small punctate vesicles can be seen in (a), and larger circular vesicles/vacuoles in which the membranes are labelled can be seen in (b-f) (white arrows). Bar, 3.0 $\mu \mathrm{m}$ vacuole lumen vital marker CDCFDA (Pringle et al., 1989) (Fig. 3d-f, arrows).

\section{Inhibitor treatments}

FM4-64 uptake was assessed in the presence of various metabolic and endocytic inhibitors (Fig. 4). The test compound was added $15 \mathrm{~min}$ prior to the addition of the FM4-64 and kept present during the course of the experiment. Using this protocol, endomembrane and vesicle labelling was inhibited by incubation of the reaction mixture at $4{ }^{\circ} \mathrm{C}$, with small punctate labelling visible. Treatment with azide/fluoride disrupted FM4-64 uptake, and resulted in diffuse cytoplasmic staining. Addition of the vacuolar marker CDCFDA also resulted in diffuse cytoplasmic labelling by the dye, verifying alterations in intracellular $\mathrm{pH}$ consistent with treatment by these metabolic poisons. These data support the idea that there is an energy-dependent step involved between uptake at discrete sites on the plasma membrane and vesicle formation (Penalva, 2005). In contrast, vesicles, albeit somewhat distorted, were still observed in germinated conidia treated with the actin polymerization inhibitor cytochalasin $\mathrm{D}(5 \mu \mathrm{M})$, although a class of smaller vesicles were noted that were not apparent in most untreated control cells. Treatment of germlings with monensin $(5 \mu \mathrm{M})$, a compound that inhibits intracellular protein transport and disrupts vesicle trafficking, resulted in decreased staining throughout the germtube, although in many cases the conidial base showed patches of strong (membrane) labelling. Vesicles were evident in most samples, although subjectively fewer were observed in treated samples as compared with controls. Latrunculin A (tested at $50 \mu \mathrm{M}$ ), a compound that interferes with F-actin, blocking endocytosis, resulted in very weak membrane staining, with little FM4-64 internalization; however, small punctate spots at the germ tube periphery or membrane could be seen. Inhibitor treatments were reversible, and when cells were washed into fresh growth medium (SDY) and allowed to recover $(4 \mathrm{~h})$ before addition of FM4-64, normal uptake was observed (data not shown).

\section{In vivo haemolymph-derived $B$. bassiana cells}

Intrahaemocoel injection of in vitro blastospores into host insects resulted in the formation of in vivo haemolymphderived yeast-like cells within 2-4 days of injection. The in vivo cells could then be isolated and separated from haemocytes and other haemolymph components by density-gradient centrifugation. A heterogeneous mixture of cell sizes, mainly lengthwise, was noted in samples of haemolymph-derived $B$. bassiana cells harvested from the tobacco budworm $H$. virescens. These cells were typically $3-$ $4 \mu \mathrm{m}$ in width with lengths ranging from 5 to $20 \mu \mathrm{m}$. The $B$. bassiana cells from $H$. virescens haemolymph were strongly labelled with FM4-64, and staining of presumed internal vesicles in a time-dependent manner was clearly evident (Fig. 5). FM4-64 labelled the membranes and septa 


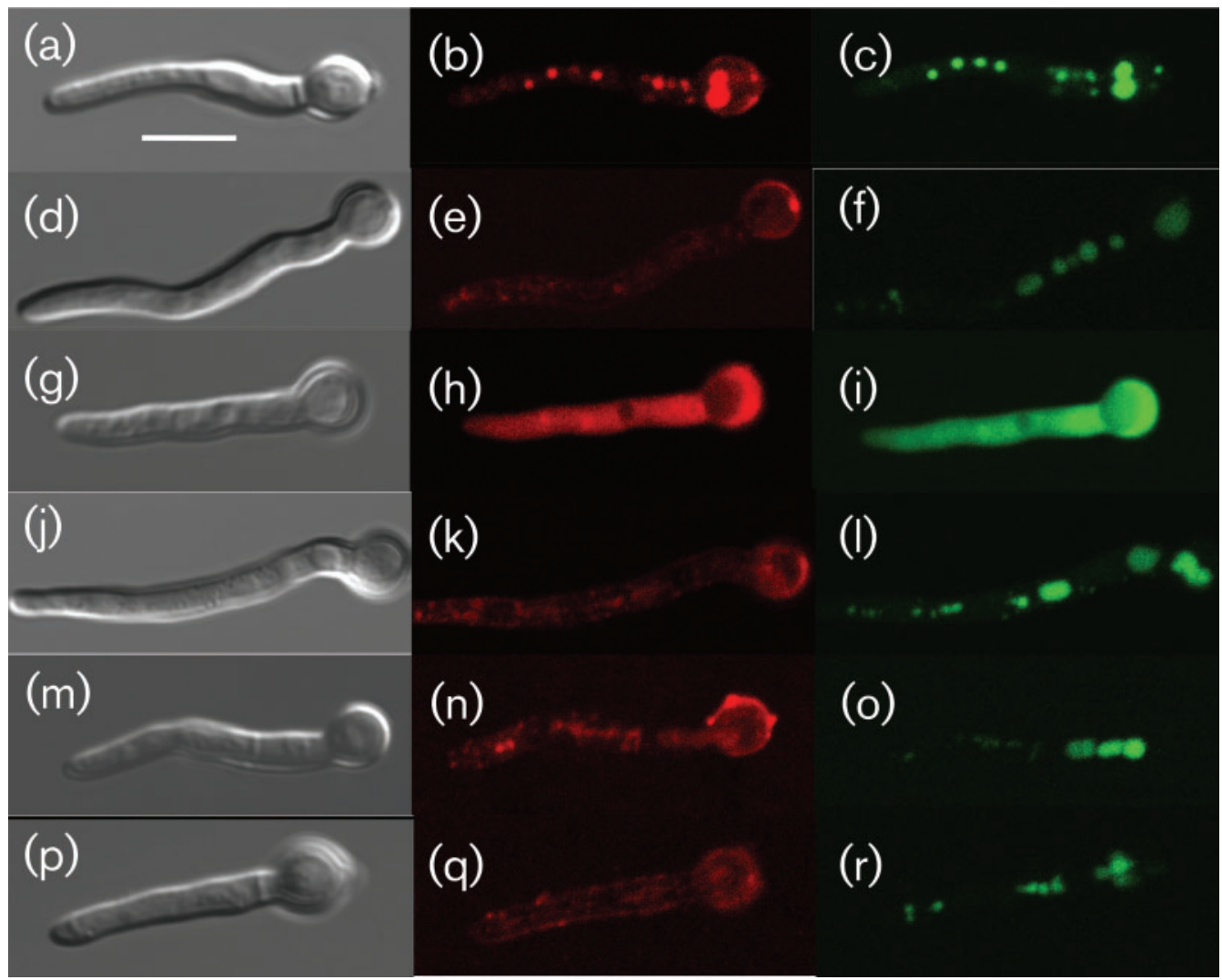

Fig. 4. Effect of treatments on FM4-64 internalization by $B$. bassiana germlings. DIC (a, d, g, j, $m$ and $p), F M 4-64$ (b, e, h, k, $n$ and $q$ ) and CDCFDA (c, $f, i, l, o$ and $r)$ visualized samples of control $(a, b, c)$ and germlings treated as follows: $4{ }^{\circ} \mathrm{C}(d, e, f)$, $5 \mathrm{mM}$ azide $+5 \mathrm{mM}$ fluoride $(\mathrm{g}, \mathrm{h}, \mathrm{i}), 5 \mu \mathrm{M}$ cytochalasin $\mathrm{D}(\mathrm{j}, \mathrm{k}, \mathrm{l}), 5 \mu \mathrm{M}$ monensin $(\mathrm{m}, \mathrm{n}, \mathrm{o})$ and $50 \mu \mathrm{M}$ latrunculin $\mathrm{A}$ ( $\mathrm{p}, \mathrm{q}, \mathrm{r})$. Bar, $5 \mu \mathrm{m}$.

of the hyphal bodies, and the time-dependent progression of labelling of small punctate structures to larger vesiclelike structures could be discerned. Some diffuse labelling of the cytoplasm was also evident. A set of large organelles, visible as dark zones in many cells, were not labelled by the dye, even after prolonged incubation (60 min). Co-staining with DAPI revealed that these organelles represented nuclei, and most of the in vivo hyphal bodies were multinucleate (Fig. $5 \mathrm{~h}-\mathrm{j}$ ). Similar to what was observed for in vitro germlings, quantification of the FM4-64 signal indicated $10-20 \%$ internalization of the dye under the conditions tested (Fig. 6). The effects of various metabolic and endocytic inhibitors on FM4-64 uptake by $H$. virescens-derived hyphal bodies was tested as described in Methods. Viability of the cells remained at $>85 \%$ under the conditions of the experiment, as determined by PI exclusion. Incubation of the uptake reaction at $4{ }^{\circ} \mathrm{C}$ resulted in lowered membrane labelling, with small punctate vesicles visible at the membrane periphery after $\sim 1 \mathrm{~h}$ (Fig. $7 \mathrm{~d}$, e and f). Treatment with azide/fluoride disrupted FM4-64 uptake, with small punctate spots (vesicles) visible. Similar to what was observed for growing hyphae, addition of CDCFDA resulted in diffuse cytoplas- mic staining (Fig. $7 \mathrm{~g}, \mathrm{~h}$ and i). Two phenotypes were observed in the presence of cytochalasin D. In several instances, clear labelling of large vacuoles (as confirmed by CDCFDA staining) was visible (Fig. 7j, $\mathrm{k}$ and $\mathrm{l}$ ), whereas in other cells, only light diffuse internal staining was evident. Treatment of the hyphal bodies with monensin resulted in FM4-64 labelling of large irregular shaped vacuoles (Fig. $7 \mathrm{~m}, \mathrm{n}$ and $\mathrm{o}$ ), whereas treatment with latrunculin A greatly diminished FM4-64 uptake, with the large clearly defined circular vacuoles that were often present in the hyphal bodies not stained by the dye.

Hyphal bodies derived from Manduca sexta contained two distinct populations of cells that were evident based upon their uptake of FM4-64 (Supplementary Fig. S1). Large cells, ranging in size from 3 to $4 \mu \mathrm{m}$ (width) to 5 and $20 \mu \mathrm{m}$ (length), were identified as the in vivo blastospores or hyphal bodies and were observed only in fungal-infected cells. In contrast to the cells isolated from $H$. virescens, these cells displayed poor (outer membrane) staining, with little uptake of the dye, although faint staining of internal vesicles was sometimes observed. Prolonged exposure to the dye $(1 \mathrm{~h})$ resulted in some increase in dye uptake in the larger in vivo hyphal bodies, but this was not consistently 


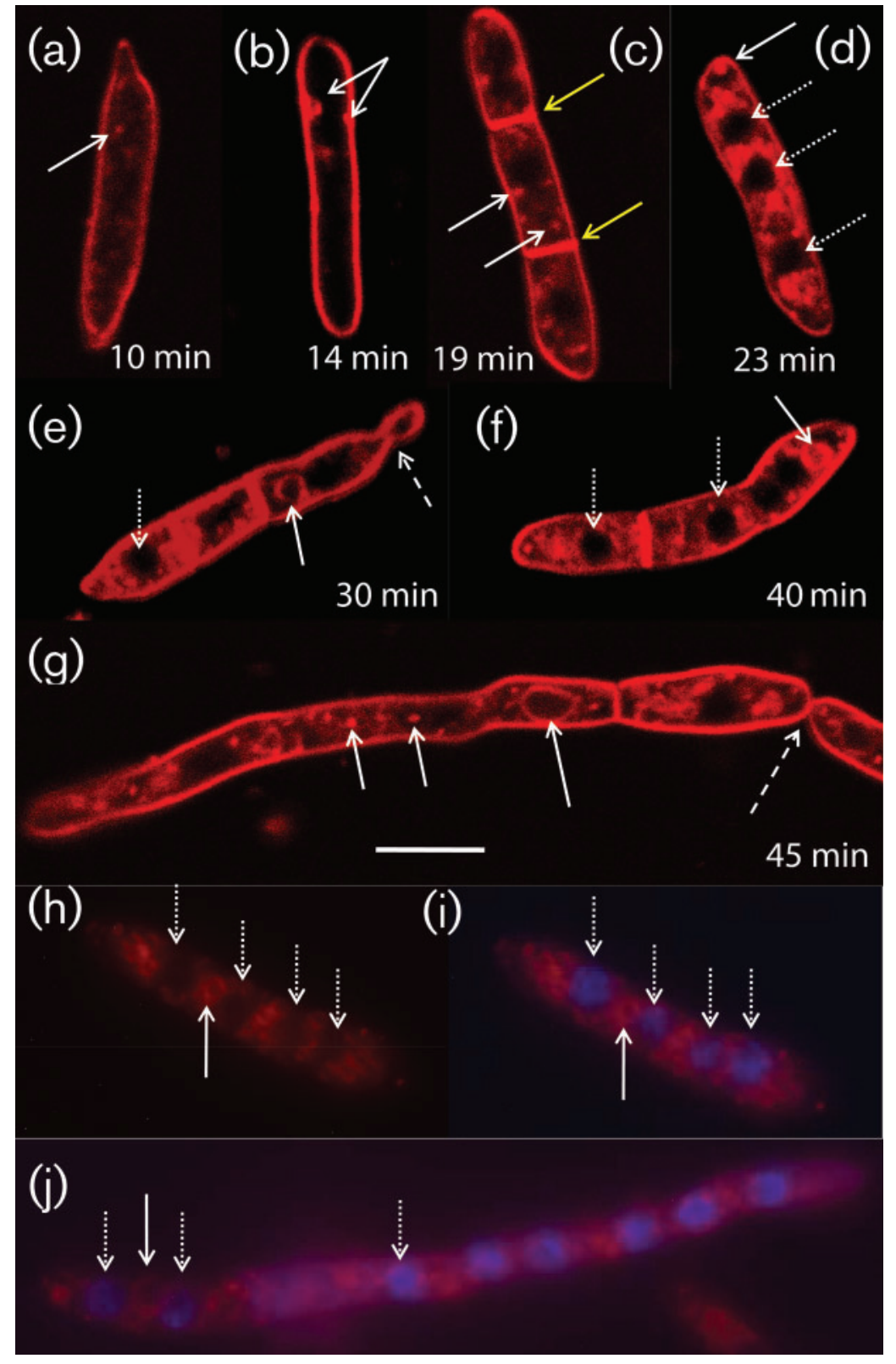

Fig. 5. FM4-64 staining of $H$. virescens haemolymph-derived in vivo hyphal bodies. In vivo cells were isolated and treated with FM464 as described in Methods. Small punctate spots (presumed vesicles) that appear as larger ones over time are shown by solid white arrows, septa are labelled with yellow arrows, regions in which the dye is absent and no discernible membrane is labelled with FM4-64 (likely nuclei, see below) are indicated by short-dashed/dotted arrows, and presumed yeast-like growth bud points are labelled with long-dashed arrows. Dual labelling using FM4$64(45 \mathrm{~min})$ followed by DAPI $(10 \mathrm{~min})(\mathrm{h}-\mathrm{j})$. Bar, $5 \mu \mathrm{m}$.

observed. No difference in viability of these cells as compared with the $H$. virescens cells was seen, and equal proportions of the cells (typically $>80 \%$ ) grew out by hyphal extension when placed into SAB medium for 8$12 \mathrm{~h}$. However, a set of smaller cells $(1-2 \mu \mathrm{m} \times 3-4 \mu \mathrm{m})$ were observed and deemed to be insect-derived, since they were seen in buffer-only-injected as well as non-injected insects. These cells displayed an intense diffuse staining under all experimental conditions tested, including room temperature loading, using the cold-load chase method, and the continuous dye treatment conditions, and appeared to have absorbed or acted as sinks for the dye, thus obscuring labelling of the fungal cells. In instances where sufficiently pure Manduca sexta-derived hyphal bodies could be recovered, FM4-64 uptake and labelling of internal vesicles could clearly be seen (Supplementary Fig. S1c, d).

\section{DISCUSSION}

The endocytic pathway has been extensively studied and described in various yeasts by both biochemical and molecular genetic studies (Gachet \& Hyams, 2005; Toret \& Drubin, 2006; Toret et al., 2008). These processes have also been examined in numerous filamentous fungi, with the primary focus on vegetative growth from spores growing by apical extension at hyphal tips (Atkinson et al., 2002; Dijksterhuis, 2003; Fischer-Parton et al., 2000). In this regard, the amphiphilic styryl dye FM4-64 has 


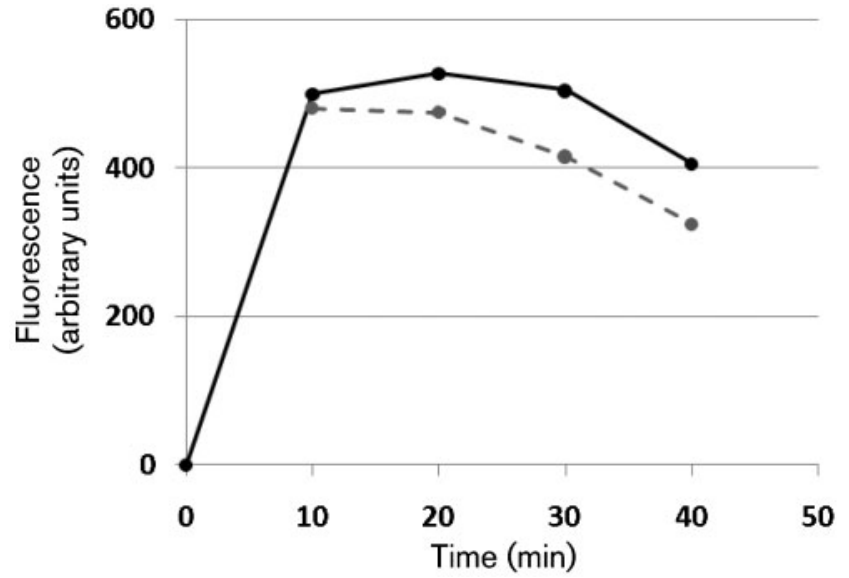

Fig. 6. Quantification of the FM4-64 signal observed from the insect haemolymph-derived hyphal bodies over time, showing total fluorescence intensity (solid line) and the membrane-only signal (dashed line).

proved to be a useful tool in examining endocytosis and vesicle trafficking in growing hyphae of a range of filamentous fungi. Studies on A. nidulans have revealed a variety of structures, including the Spitzenkörper, cortical punctuate organelles, mature endosome/vacuoles, and larger vacuoles (whose lumens are strongly labelled with CDCFDA), as well as portions of the mitochondrial network and even (weakly) the nuclear membrane (Penalva, 2005). Mutants and enhanced GFP (eGFP)tagged rescue of the vacuolar syntaxin in $A$. orzyae, examined by FM4-64 staining, have revealed syntaxin localization on the membrane of pleomorphic vacuolar networks that include large spherical and tubular vacuoles, as well as putative late-endosomal compartments (Shoji et al., 2006). More recently, it has been shown that various components of the endocytic machinery of $A$. nidulans appear to be preferentially localized to actin cytoskeletal polarization near the hyphal tips, which has led to a model in which endocytosis plays a role in cell polarity and apical membrane recycling (Araujo-Bazan et al., 2008; Higuchi et al., 2009). For the plant pathogen U. maydis, tip growth and pathogenicity have been shown to involve essential endocytic recycling pathways, with bidirectional movement of vesicles mediated by various molecular motors (Steinberg, 2007).

B. bassiana is unique in that it produces at least three distinct in vitro single-cell propagules, namely aerial conidia, blastospores (in vitro) and submerged conidia, as well as specialized in vivo haemolymph-residing cells during insect infection (Hegedus et al., 1992; Pendland et al., 1993). The in vitro cells are morphologically and biochemically distinct and appear to have stage-specific gene expression profiles (Cho et al., 2006b; Holder \& Keyhani, 2005; Holder et al., 2007). In this report, we have examined FM4-64 uptake in germinating conidia growing by hyphal extension, and in a number of in vitro and insect haemolymph-derived cell populations. Little to no internalization of the FM4-64 dye was observed in aerial conidia, which likely indicates the dormancy of the cells rather than the lack of endocytic mechanisms per se. Internalization was evident for both in vitro blastospores and submerged conidia, although discrete vesicles and/or internal structures were difficult to discern. In contrast, for germlings derived from germinated conidia, incorporation of the dye followed a distinct sequence of events, beginning with staining of cortical punctate structures or spots, possibly representing actin patches, which were distributed along hyphae and basal conidiophores. Staining of the punctate structures was followed by staining of the membranes of small $(0.5-1.0 \mu \mathrm{m})$, circular organelles that were similar to reported 'late' pre-vacuolar endosomes (Fischer-Parton et al., 2000; Penalva, 2005). Finally, over time, staining of the membranes of larger organelles (1$3 \mu \mathrm{m}$ ), representing vacuoles as confirmed by CDCFDA co-staining, were identified. The largest vacuoles in any given germling were usually located at or close to the basal conidiospore compartment, and vacuole diameter appeared to decrease with increased distance from the growing hyphal tip. Treatment of germlings with azide/ fluoride appeared to arrest staining at the cortical punctate stage, indicating the possible requirement of energy for early endosome formation. Latrunculin B treatment greatly inhibited FM4-64 internalization, and in contrast to the $A$. nidulans findings (Penalva, 2005), inhibited staining of the plasma membrane. Cytochalasin D and monensin treatment resulted in lower overall uptake, with decreased vesicle staining, mainly limited to the larger vacuoles. No internalization of the LYCH dye was observed for any of the B. bassiana cells tested; however, such a lack of uptake has been reported in other fungi (Atkinson et al., 2002; Read \& Kalkman, 2003). These results may reflect nonproductive interactions between the dye and the membranes or the fact that only small amounts of the fluid phase and hence of $\mathrm{LYCH}$ are internalized, implying that the role of endocytosis is the turnover of membrane and membrane-associated protein rather than internalization of vesicle components and nutrient uptake.

B. bassiana also has a parasitic life cycle. Once bound to an insect cuticle, fungal conidia germinate to form germ tubes that digest and penetrate the insect cuticle and subsequently the integument, reaching the haemocoel. Once inside the insect haemolymph, the fungus produces yeastlike in vivo cells that are morphologically and biochemically distinct from the in vitro cells, and which are known to produce unique in vivo (anti-insect) toxins (Mazet et al., 1994; Pendland et al., 1993; Tartar \& Boucias, 2004). The in vivo blastospores or hyphal bodies were somewhat heterogeneous, particularly in length, ranging from 3 to $4 \mu \mathrm{m}$ (width) to 5 and $20 \mu \mathrm{m}$ (length). Hyphal bodies isolated from Manduca sexta appeared recalcitrant to dye uptake, often appearing as haloes in images; however, this was likely due to the presence of contaminating insect- 


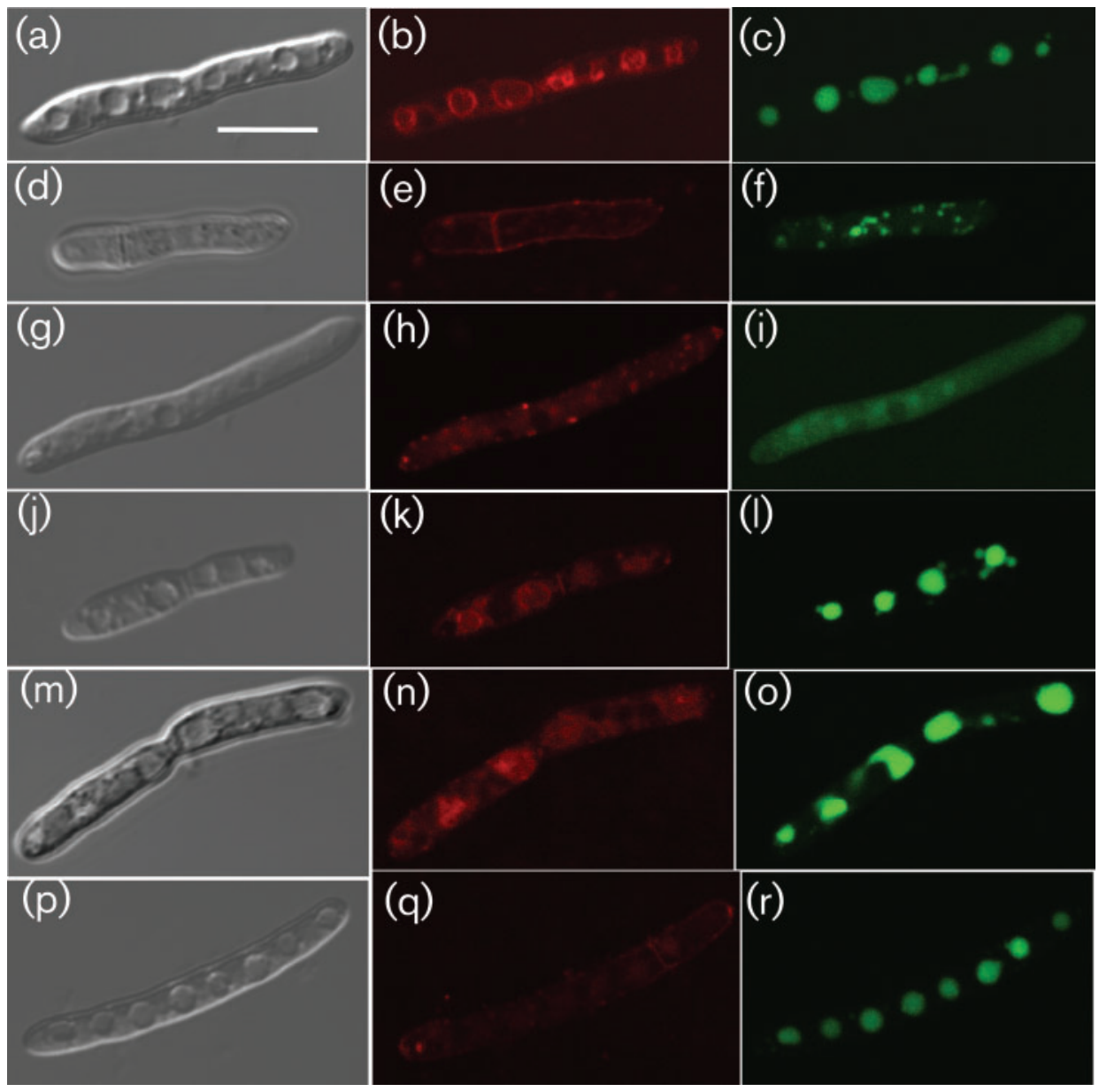

Fig. 7. Effect of treatments on FM4-64 internalization by $H$. virescens-derived $B$. bassiana hyphal bodies. DIC (a, d, g, j, m and p), FM4-64 (b, e, h, k, n and q) and CDCFDA (c, f, i, l, o and r) visualized samples of control (a, b, c) and germlings treated as follows: $4{ }^{\circ} \mathrm{C}(\mathrm{d}, \mathrm{e}, \mathrm{f}), 5 \mathrm{mM}$ azide $+5 \mathrm{mM}$ fluoride $(\mathrm{g}, \mathrm{h}, \mathrm{i}), 5 \mu \mathrm{M}$ cytochalasin $\mathrm{D}(\mathrm{j}, \mathrm{k}, \mathrm{l}), 5 \mu \mathrm{M}$ monensin $(\mathrm{m}, \mathrm{n}, \mathrm{o})$ and $50 \mu \mathrm{M}$ latrunculin $A(p, q, r)$. Bar, $5 \mu \mathrm{m}$.

derived cells that appeared to sequester the dye, and in some instances after prolonged exposure to the dye, cortical punctate staining could be seen. Furthermore, in samples with apparently greater purity of the hyphal bodies, membrane and vesicle staining could clearly be detected. In contrast to the Manduca sexta-derived hyphal bodies, $B$. bassiana cells isolated from $H$. virescens haemolymph actively transported the FM4-64 dye, with membrane staining of small and large vesicles consistently observed. Disruption of dye uptake occurred when reactions were incubated at $4{ }^{\circ} \mathrm{C}$ and in the presence of azide/fluoride. Internal vesicle staining was evident after treatment of the hyphal bodies with cytochalasin D and monensin, although distortions in the vesicles themselves were apparent. Similar to what was seen for growing hyphae, treatment of the haemolymph-derived cells with Latrunculin A appeared to significantly diminish FM4-64 uptake. Overall, our data support the idea that active endocytosis occurs in cells that have differentiated into the freely circulating in vivo haemolymph stage. Additional experiments examining the underlying reasons for the variation in FM4-64 uptake in the different B. bassiana cell types and what role the observed phenomenon may represent in the development, host immune interaction and nutrient uptake of the haemolymph-derived cells are warranted, particularly since it is becoming increasingly clear that endocytosis is not limited to a role in nutrient uptake but likely plays a role in cell growth and shape via maintenance of specific membrane-bound proteins, subdomains and structures (Lee et al., 2008; Steinberg, 2007). Indeed, the lack of LYCH uptake may imply that the role of endocytosis is membrane and membrane-associated protein turnover rather than internalization of vesicle components and nutrient uptake.

With advances in genomics, the underlying mechanisms of polarized growth and hyphal morphogenesis in filamentous fungi are increasingly coming under molecular scrutiny 
(Harris et al., 2005; Virag \& Harris, 2006). Knowledge concerning these processes in entomopathogenic fungi, particularly within the context of host-pathogen interactions, remains limited. The recent availability of an extensive expressed sequence tag (EST) dataset for $B$. bassiana, coupled to methods for examining differential gene regulation and constructing gene knockouts, should allow for a detailed molecular dissection of these pathways that is likely to lead to novel insights into developmental programs and nutrient uptake in (pathogenic) filamentous fungi (Cho et al., 2006a, b; Eley et al., 2007; Pathan et al., 2007).

\section{REFERENCES}

Araujo-Bazan, L., Penalva, M. A. \& Espeso, E. A. (2008). Preferential localization of the endocytic internalization machinery to hyphal tips underlies polarization of the actin cytoskeleton in Aspergillus nidulans. Mol Microbiol 67, 891-905.

Atkinson, H. A., Daniels, A. \& Read, N. D. (2002). Live-cell imaging of endocytosis during conidial germination in the rice blast fungus, Magnaporthe grisea. Fungal Genet Biol 37, 233-244.

Ayscough, K. R. (2005). Defining protein modules for endocytosis. Cell 123, 188-190.

Cho, E. M., Boucias, D. \& Keyhani, N. O. (2006a). EST analysis of cDNA libraries from the entomopathogenic fungus Beauveria (Cordyceps) bassiana. II. Fungal cells sporulating on chitin and producing oosporein. Microbiology 152, 2855-2864.

Cho, E. M., Liu, L., Farmerie, W. \& Keyhani, N. O. (2006b). EST analysis of cDNA libraries from the entomopathogenic fungus Beauveria (Cordyceps) bassiana. I. Evidence for stage-specific gene expression in aerial conidia, in vitro blastospores and submerged conidia. Microbiology 152, 2843-2854.

Cole, L., Hyde, G. J. \& Ashford, A. E. (1997). Uptake and compartmentalisation of fluorescent probes by Pisolithus tinctorius hyphae: evidence for an anion transport mechanism at the tonoplast but not for fluid-phase endocytosis. Protoplasma 199, 18-29.

Dijksterhuis, J. (2003). Confocal microscopy of Spitzenkorper dynamics during growth and differentiation of rust fungi. Protoplasma 222, 53-59.

Dimbi, S., Maniania, N. K., Lux, S. A., Ekesi, S. \& Mueke, J. K. (2003). Pathogenicity of Metarhizium anisopliae (Metsch.) Sorokin and Beauveria bassiana (Balsamo) Vuillemin, to three adult fruit fly species: Ceratitis capitata (Weidemann), C. rosa var. fasciventris Karsch and C. cosyra (Walker) (Diptera:Tephritidae). Mycopathologia 156, 375-382.

Eley, K. L., Halo, L. M., Song, Z. S., Powles, H., Cox, R. J., Bailey, A. M., Lazarus, C. M. \& Simpson, T. J. (2007). Biosynthesis of the 2-pyridone tenellin in the insect pathogenic fungus Beauveria bassiana. ChemBioChem 8, 289-297.

Fischer-Parton, S., Parton, R. M., Hickey, P. C., Dijksterhuis, J., Atkinson, H. A. \& Read, N. D. (2000). Confocal microscopy of FM4-64 as a tool for analysing endocytosis and vesicle trafficking in living fungal hyphae. Journal of Microscopy 198, 246-259.

Fuchs, U., Hause, G., Schuchardt, I. \& Steinberg, G. (2006). Endocytosis is essential for pathogenic development in the corn smut fungus Ustilago maydis. Plant Cell 18, 2066-2081.

Gachet, Y. \& Hyams, J. S. (2005). Endocytosis in fission yeast is spatially associated with the actin cytoskeleton during polarised cell growth and cytokinesis. J Cell Sci 118, 4231-4242.
Gurunathan, S., Marash, M., Weinberger, A. \& Gerst, J. E. (2002). tSNARE phosphorylation regulates endocytosis in yeast. Mol Biol Cell 13, 1594-1607.

Harris, S. D., Read, N. D., Roberson, R. W., Shaw, B., Seiler, S., Plamann, M. \& Momany, M. (2005). Polarisome meets Spitzenkorper: microscopy, genetics, and genomics converge. Eukaryot Cell 4, 225229.

Hegedus, D. D., Bidochka, M. J., Miranpuri, G. S. \& Khachatourians, G. G. (1992). A comparison of the virulence, stability, and cell-wallsurface characteristics of three spore types produced by the entomopathogenic fungus Beauveria bassiana. Appl Microbiol Biotechnol 36, 785-789.

Hickey, P. C., Jacobson, D. J., Read, N. D. \& Glass, N. L. (2002). Livecell imaging of vegetative hyphal fusion in Neurospora crassa. Fungal Genet Biol 37, 109-119.

Higuchi, Y., Shoji, J. Y., Arioka, M. \& Kitamoto, K. (2009). Endocytosis is crucial for cell polarity and apical membrane recycling in the filamentous fungus Aspergillus oryzae. Eukaryot Cell 8, 37-46.

Holder, D. J. \& Keyhani, N. O. (2005). Adhesion of the entomopathogenic fungus Beauveria (Cordyceps) bassiana to substrata. Appl Environ Microbiol 71, 5260-5266.

Holder, D. J., Kirkland, B. H., Lewis, M. W. \& Keyhani, N. O. (2007). Surface characteristics of the entomopathogenic fungus Beauveria (Cordyceps) bassiana. Microbiology 153, 3448-3457.

Inglis, G. D., Goettel, M. S., Butt, T. M. \& Strasser, H. (2001). Use of hyphomycetous fungi for managing insect pests. In Field Manual of Techniques in Invertebrate Pathology, pp. 651-679. Edited by L. A. Lacey \& H. K. Kaya. Dordrecht, The Netherlands: Kluwer Academic.

Kaksonen, M., Sun, Y. \& Drubin, D. G. (2003). A pathway for association of receptors, adaptors, and actin during endocytic internalization. Cell 115, 475-487.

Kassa, A., Stephan, D., Vidal, S. \& Zimmermann, G. (2004). Production and processing of Metarhizium anisopliae var. acridum submerged conidia for locust and grasshopper control. Mycol Res 108, 93-100.

Kirkland, B. H., Westwood, G. S. \& Keyhani, N. O. (2004). Pathogenicity of entomopathogenic fungi Beauveria bassiana and Metarhizium anisopliae to Ixodidae tick species Dermacentor variabilis, Rhipicephalus sanguineus, and Ixodes scapularis. J Med Entomol 41, 705-711.

Lecuona, R. E., Turica, M., Tarocco, F. \& Crespo, D. C. (2005). Microbial control of Musca domestica (Diptera: Muscidae) with selected strains of Beauveria bassiana. J Med Entomol 42, 332-336.

Lee, S. C., Schmidtke, S. N., Dangott, L. J. \& Shaw, B. D. (2008). Aspergillus nidulans ArfB plays a role in endocytosis and polarized growth. Eukaryot Cell 7, 1278-1288.

Lomer, C. J., Bateman, R. P., Johnson, D. L., Langewald, J. \& Thomas, M. (2001). Biological control of locusts and grasshoppers. Annu Rev Entomol 46, 667-702.

Mazet, I., Hung, S. Y. \& Boucias, D. G. (1994). Detection of toxic metabolites in the hemolymph of Beauveria bassiana infected Spodoptera exigua larvae. Experientia 50, 142-147.

McCoy, C. W. (1990). Entomogenous fungi as microbial pestidides. In New Directions in Biological Control, pp. 139-159. Edited by R. R. Baker \& P. E. Dunn. New York: A.R. Liss.

Murphy, A. S., Bandyopadhyay, A., Holstein, S. E. \& Peer, W. A. (2005). Endocytotic cycling of PM proteins. Annu Rev Plant Biol 56, 221-251.

Pathan, A. A. K., Devi, K. U., Vogel, H. \& Reineke, A. (2007). Analysis of differential gene expression in the generalist entomopathogenic fungus Beauveria bassiana (Bals.) Vuillemin grown on different insect 
cuticular extracts and synthetic medium through cDNA-AFLPs. Fungal Genet Biol 44, 1231-1241.

Penalva, M. A. (2005). Tracing the endocytic pathway of Aspergillus nidulans with FM4-64. Fungal Genet Biol 42, 963-975.

Pendland, J. C., Hung, S. Y. \& Boucias, D. G. (1993). Evasion of host defense by in vivo-produced protoplast-like cells of the insect mycopathogen Beauveria bassiana. J Bacteriol 175, 5962-5969.

Pringle, J. R., Preston, R. A., Adams, A. E. M., Stearns, T., Drubin, D. G., Haarer, B. K. \& Jones, E. W. (1989). Fluorescence microscopy methods for yeast. Methods Cell Biol 31, 357-435.

Read, N. D. \& Kalkman, E. R. (2003). Does endocytosis occur in fungal hyphae? Fungal Genet Biol 39, 199-203.

Rehner, S. A. \& Buckley, E. (2005). A Beauveria phylogeny inferred from nuclear ITS and EF1-alpha sequences: evidence for cryptic diversification and links to Cordyceps teleomorphs. Mycologia 97, 84-98.

Samson, R. A., Evans, C. S. \& Latgé, J. P. (1988). Atlas of Entomopathogenic Fungi. Berlin: Springer-Verlag.

Scholte, E. J., Knols, B. G. J., Samson, R. A. \& Takken, W. (2004). Entomopathogenic fungi for mosquito control: a review. J Insect Sci 4, 19.

Shoji, J. Y., Arioka, M. \& Kitamoto, K. (2006). Vacuolar membrane dynamics in the filamentous fungus Aspergillus oryzae. Eukaryot Cell 5, 411-421.

Smythe, E. \& Ayscough, K. R. (2006). Actin regulation in endocytosis. J Cell Sci 119, 4589-4598.

Steinberg, G. (2007). On the move: endosomes in fungal growth and pathogenicity. Nat Rev Microbiol 5, 309-316.
Tartar, A. \& Boucias, D. G. (2004). A pilot-scale expressed sequence tag analysis of Beauveria bassiana gene expression reveals a tripeptidyl peptidase that is differentially expressed in vivo. Mycopathologia 158, 201-209.

Thomas, K. C., Khachatourians, G. G. \& Ingledew, W. M. (1987). Production and properties of Beauveria bassiana conidia cultivated in submerged culture. Can J Microbiol 33, 12-20.

Toret, C. P. \& Drubin, D. G. (2006). The budding yeast endocytic pathway. J Cell Sci 119, 4585-4587.

Toret, C. P., Lee, L., Sekiya-Kawasaki, M. \& Drubin, D. G. (2008). Multiple pathways regulate endocytic coat disassembly in Saccharomyces cerevisiae for optimal downstream trafficking. Traffic 9, 848-859.

Torralba, S. \& Heath, I. B. (2002). Analysis of three separate probes suggests the absence of endocytosis in Neurospora crassa hyphae. Fungal Genet Biol 37, 221-232.

Vida, T. A. \& Emr, S. D. (1995). A new vital stain for visualizing vacuolar membrane dynamics and endocytosis in yeast. $J$ Cell Biol 128, 779-792.

Virag, A. \& Harris, S. D. (2006). The Spitzenkorper: a molecular perspective. Mycol Res 110, 4-13.

Wanchoo, A., Lewis, M. W. \& Keyhani, N. O. (2009). Lectin mapping reveals stage-specific display of surface carbohydrates in in vitro and haemolymph-derived cells of the entomopathogenic fungus Beauveria bassiana. Microbiology 155, 3121-3133.

Edited by: B. A. Horwitz 